\title{
Intermédialités
}

Histoire et théorie des arts, des lettres et des techniques

Intermediality

History and Theory of the Arts, Literature and Technologies

\section{Intermédialités : le temps des illusions perdues}

\section{Éric Méchoulan}

Numéro 1, printemps 2003

Naître

URI : https://id.erudit.org/iderudit/1005442ar

DOI : https://doi.org/10.7202/1005442ar

Aller au sommaire du numéro

Éditeur(s)

Centre de recherche sur l'intermédialité

ISSN

1920-3136 (numérique)

Découvrir la revue

Citer cet article

Méchoulan, É. (2003). Intermédialités : le temps des illusions perdues.

Intermédialités / Intermediality, (1), 9-27. https://doi.org/10.7202/1005442ar

\section{Résumé de l'article}

Après l'intertextualité qui visait à sortir le texte de son autonomie supposée; après l'interdiscursivité qui saisissait que l'unité est constituée des multiples discours que ramasse et traverse le texte; voici l'intermédialité qui étudie comment textes et discours ne sont pas seulement des ordres de langage, mais aussi des supports, des modes de transmission, des apprentissages de codes, des leçons de choses. Ces médiations ont des histoires : les intermédialités en forment les plans de consistance. Le concept d'intermédialité opère alors à trois niveaux différents d'analyse. Il peut désigner, d'abord, les relations entre divers médias (voire entre diverses pratiques artistiques associées à des médias délimités). Ensuite, ce creuset de média d'où émerge et s’institutionnalise peu à peu un médium bien circonscrit. Enfin, le milieu en général dans lequel les médias prennent forme et sens : l'intermédialité est immédiatement présente à toute pratique d'un médium. Encore faut-il en percevoir aussi l'actualité en fonction des nouvelles figures sociales du travail immatériel. 


\title{
Intermédialités : \\ Le temps des illusions perdues
}

\author{
É R I C M É C H O U L A N
}

«Ce qui est ferme, est par le temps destruit,

Et ce qui fuit, au temps fait resistance. »

JoAchim Du Bellay, Antiquitez de Rome, III

omme tous les concepts qui sonnent terriblement à la mode, l'intermédialité doit faire naître les soupçons plus que les enthousiasmes et l'interrogation plus que l'adoption automatique. Rien de nouveau sous le soleil qui ne soit susceptible d'une illusoire séduction. Dans chaque Maintenant, il en va des fausses vérités ou des fausses sciences comme de la fausse monnaie selon la loi de Gresham: elles l'emportent sur la vraie. Il est donc important de commencer par un examen soigneux de ce qui préside à sa naissance, afin d'en éviter les dérives possibles ou d'en relever les avantages à exploiter.

À l'évidence, cette «intermédialité» suppose d'abord l'enchaînement de diverses théories qui ont, elles aussi, joué des valeurs de ce même préfixe. Ainsi, l'intertextualité visait à sortir le texte de son autonomie supposée et à lire en lui la mise en œuvre d'autres textes préexistants, le restituant à une chaîne d'énoncés et mesurant ce qu'il devait à des œuvres antérieures - voire postérieures: plagiat par anticipation, comme Raymond Roussel et certaines pratiques de l'oulipo, ou reprise modifiant la perception et l'interprétation d'ouvrages préexistants, comme lorsque Bacon repeint le portrait du pape Innocent X de Vélasquez ou que JeanLuc Godard filme Prénom: Carmen (1983). Par «texte», on comprend qu'il faut entendre toute production esthétique qui constitue un «tissu» de mots, de sons, de pigments ou d'images: ainsi, l'iconologie panofskienne est, bien avant les intertextualités mises à la mode par la critique littéraire des années soixante et soixante-dix, la recherche de motifs ou de dispositifs iconiques qui essaiment de toile en 
toile. Ces enquêtes demeurent pourtant dans l'homogénéité d'une tradition, qu'elle soit littéraire ou picturale. Or, l'ordre des œuvres ne dépend pas seulement de la sphère institutionnelle à laquelle ils appartiennent, mais aussi des multiples « discours » et «représentations» qui s’y trouvent ramassés, tressés, traversés: discours quotidiens, discours des disciplines ou des champs de compétence, voire plus largement formations discursives qui articulent le représentable à des représentés historiquement circonscrits. Chaque ouvrage suppose alors la performance de diverses compétences dont doit rendre compte une «interdiscursivité».

Et l'intermédialité? Elle ouvre sur un champ encore plus large ou encore plus fondamental, car elle observe qu'une ouvre ne fonctionne pas seulement dans ses dettes plus ou moins reconnues envers telles autres œuvres, ou dans la mobilisation de compétences discursives (au besoin usurpées), mais également dans le recours à des institutions qui en permettent l'efficacité et à des supports matériels qui en déterminent l'effectivité. Dans les discours, on ne trouve donc pas simplement des paroles neutralisées dans des champs de compétence (qui sont des régimes spécifiques d'autorité), mais encore deux modes de «support»: par en haut, des institutions socialement reconnues, par en bas, des matières techniquement ouvragées - et encore est-ce déjà beaucoup s'avancer selon des usages préfabriqués que d'assigner de la sorte la «hauteur» aux institutions et la «bassesse» aux techniques et aux matériaux: semblable jugement de valeur dépend lui aussi d'une organisation sociale de la pensée. Il n'en demeure pas moins que l'efficacité orchestrée par les institutions et l'effectivité induite par les techniques et les matériaux produisent, au bout du compte, des effets de sens.

L'intermédialité étudie donc comment textes, images et discours ne sont pas seulement des ordres de langage ou de symbole, mais aussi des supports, des modes de transmission, des apprentissages de codes, des leçons de choses. Ces matérialités de la communication font partie du travail de signification et de référence, de même que les productions symboliques, les Idées ne flottent pas dans un éther insondable ou ne sont pas seulement des constructions spirituelles étrangères à leurs composantes concrètes. Les effets de sens sont aussi des dispositifs sensibles. Cela ne signifie pas que la production de sens serait réductible à des procédures du sensible, mais simplement qu'il existe des liens entre le sens du sensible et le sens du sensé, entre le physique (ou la matière) et le sémantique (ou l'idée). En fait, on observe que l'insistance contemporaine sur l'intertextualité, l'interdiscursivité et, maintenant, l'intermédialité repose sur un principe de continuité entre des ordres de plus en plus distants les uns des autres: d'abord, d'un texte avec divers textes du même champ; ensuite, d'un texte avec des discours de 
multiples champs; enfin, d'un discours avec des performances discursives, des supports institutionnels et sensibles hétérogènes. Pour le dire autrement, il semble qu'à chaque fois c'est le principe d'une coupure ou d'une autonomie qui est remis en cause: d'un texte par rapport à d'autres textes, d'un texte par rapport à un contexte discursif, d'un discours par rapport aux forces sociales des institutions ou aux forces physiques des techniques et des matériaux. Bref, le préfixe «inter» vise à mettre en évidence un rapport inaperçu ou occulté, ou, plus encore, à soutenir l'idée que la relation est par principe première : là où la pensée classique voit généralement des objets isolés qu'elle met ensuite en relation, la pensée contemporaine insiste sur le fait que les objets sont avant tout des nœuds de relations, des mouvements de relation assez ralentis pour paraître immobiles.

Dans cet enchaînement de l'intertextualité à l'interdiscursivité puis à l'intermédialité, j'ai fait comme si, d'une part, «inter» occupait une fonction neutre, et, d'autre part, textes, discours et médias jouaient au même niveau. Bien sûr, il n'en est rien. Il faut donc examiner de plus près ces deux composantes et préciser leurs implications.

Commençons par le préfixe et revenons à son étymologie (non comme à sa vérité, mais comme aux possibilités de pensée qu'elle offre): le latin inter désigne le fait de se trouver au milieu de deux éléments, qu'ils soient spatiaux (entre deux chaises) ou temporels (inter noctem, par exemple, signifie «pendant la nuit»). Le fait d' «être-entre» (inter-esse) consiste donc à se trouver au milieu de deux instances; cependant, le verbe ne désigne pas simplement la distance de deux lieux, mais aussi leur différence, en particulier dans la forme impersonnelle interest qui est à la fois descriptive (ceci est différent de cela, du point de vue de la quantité, pour ainsi dire) et, surtout, évaluative (ceci vaut plus que cela, du point de vue dès lors qualitatif: interest est traduit alors par «il importe que» ou «il est dans notre intérêt de»). Le verbe latin offre encore une autre dimension significative: il désigne souvent le fait même d'être présent, non en soi, mais justement dans une relation: participer à un événement, se trouver parmi des gens. Interesse suppose donc une présence aux autres, voire la présence première des autres qui donne au sujet sens et valeur dans l'événement qui les réunit. L'être-entre serait donc ce qui produit de la présence, des valeurs comparées entre les personnes ou les objets mis en présence, ainsi que des différences matérielles ou idéelles entre ces personnes ou ces objets présentés.

Cette production de présence ne conduit pas à une impeccable adéquation à soi ou à une superbe transparence de l'instant, puisque c'est en tant que différence que cette présence est produite, à commencer donc par la différence d'avec 
soi-même: le milieu, l'entre-deux est ce qui fait apparaître les extrêmes comme différence de situation et différence de valeur. Cette présence est alors un partage, au double sens du terme: ici, les différences sont mises en commun et senties ou pensées comme réciproques, là, les différences éloignent et séparent selon des lignes de divergence évidentes. Ce partage est justement un partage des sens, car le sens ne devient sensible que dans cette bifurcation qui scinde et unit l'instant présent à lui-même'.

Prenons deux cas de figure afin d'éclairer cette question.

D’abord, le cas du signe. Un signe est simultanément une trace sensible qui, littéralement, tombe sous le sens et apparaît pour elle-même, et un renvoi à un objet du monde, à un affect ou à un concept forcément différent de ce qu'il est matériellement (de même que le concept de chien n'a jamais mordu personne, un signe ne désigne autre chose que lui-même qu'en renonçant à sa signature, en se «dé-signant», en se faisant oublier comme matérialité sensible). Le bon fonctionnement du signe suppose donc à la fois que la trace apparaisse comme trace et qu'elle disparaisse sous la chose désignée. Quand on dit «regardez! » en pointant du doigt vers la porte, ceux qui regardent le doigt au lieu de tourner la tête vers la porte prêtent attention à la trace sensible de la désignation tandis que les autres oublient le doigt pour mieux focaliser leur attention sur l'objet ou l'événement indiqué.

Ensuite, un second cas de figure: le fonctionnement du présent. Si le présent n'est que ce point toujours vacillant entre passé et futur, aussitôt disparu qu'apparu dans l'instant qui lui succède, comment fait-il pour devenir passé? Comment le présent passe-t-il dans ce qu'il n'est pas (le passé)? Il faut, en fait, supposer, selon certaines formules bergsoniennes, une bifurcation intérieure à

1. On peut rapprocher cette réflexion de celle de Jean-Luc Nancy: «Il n’y a pas de sens si le sens n'est pas partagé, et cela, non pas parce qu'il y aurait une signification ultime ou première, que tous les étants auraient en commun, mais parce que le sens est luimême partage de l'être. Le sens commence là où la présence n'est pas pure présence, mais se disjoint pour être elle-même en tant que telle. Cet "en tant que" suppose écartement, espacement et partition de la présence. Le seul concept de "présence" contient la nécessité de cette partition. La pure présence impartagée, présence à rien, de rien, pour rien, n'est ni présente ni absente: simple implosion sans trace d'un être qui n'aurait jamais été. [...] Ce qui s'énonce encore de cette manière : l'être ne peut être qu'étant-les-uns-avec-lesautres, circulant dans l'avec et comme l'avec de cette co-existence singulièrement plurielle.» Etre singulier pluriel, Paris, Galilée, 1996, p. 20-21. 
chaque présent: au présent immédiat qui s'évanouit dans l'éphémère répond un présent, lui aussi immédiat (et non médiat), qui s'épanouit dans la durée. On conçoit donc que la «production de présence » n’implique pas qu'en deçà ou audelà du spectacle des apparences sensibles résiderait une Présence plus essentielle, car le présent qui dure coexiste, dans le même plan d'immanence (donc sans transcendance), avec le présent qui fuit et c'est bien leur différence même qui fait la présence du présent: c'est pourquoi il faut, à chaque fois, parler d'immédiateté.

Ainsi, contrairement à ce que l'on pourrait croire a priori, l'intermédialité a affaire avant tout à l'immédiat plutôt qu'aux médias et aux médiations - mais en considérant qu'il s'agit toujours d'effets d'immédiateté. Il y a en fait deux immédiatetés: l'une, plus simple que les médiations, qui ouvre sur la bêtise de l'instantané ou de l'intensité de ce qui meurt; l'autre, plus complexe que les médiations, à partir de laquelle se mettent en place les rapports de puissance qui forment et configurent chaque situation, chaque Maintenant, et engendrent l'intensité de ce qui vit. Ces rapports de puissance font bien comprendre qu'aucune neutralité ontologique de la Présence ne peut apparaître, sinon sous la guise d'un leurre ou d'un besoin. Les rapports de puissance sont rapports du présent à lui-même et impliquent une histoire: non seulement compte-rendu des médiations du passé, mais écriture des bifurcations du présent. En ce sens, l'historiographie se conçoit moins comme science des représentations et des résultats, plus comme art des situations et des problèmes². Situations et problèmes qui sont déterminés par les effets de valeur qui font les rapports de puissance: inter est, il importe que... - l'être-entre est toujours intéressé, car il n'existe que dans le profit de ses échanges ${ }^{3}$ (même par le don). Inter est donc loin d'être un préfixe transparent et neutre. Il nous impose de penser l'intermédialité à partir de calculs et d'évaluations, de situations et de rapports de puissance, de fabrication de présence et d'effets d'immédiateté.

2. Sur ces questions, je me permets de renvoyer à mon ouvrage à paraître De l'immédiateté, Paris, Presses universitaires de France, coll. «L'interrogation philosophique», 2003.

3. «Le devenir n'est doté de sens (ou rapporté à un sens) que là où il y va de quelque chose pour quelqu'un, là où l'on se trouve en présence de déroulements qui ne sont pas purement et simplement à constater, mais compréhensibles à partir d'un intérêt et d'un rapport au monde, d'une ouverture à soi-même et aux choses. Les premiers signes d'intérêt sont à relever avec l'apparition de la vie », Jan Patočka, «Le commencement de l'histoire», Essais hérétiques sur la philosophie de l'histoire, trad. Erika Abrams, Lagrasse, Verdier, 1999, p. 48. 
Si le temps se déroulait selon le simple schéma linéaire de l'avant et de l'après, le présent ne serait que cet entre temps qui échapperait sans cesse à soimême. Or, le présent doit plutôt se concevoir sous la forme anachronique d'un retour ou d'un pli du temps (ana-chronos, c'est un temps qui revient). L'anachronisme n'est pas le savoir de ce qui existe déjà et qui n’offre donc aucune surprise, c'est au contraire le moment où le passé me surprend parce que seul le passé est véritablement nouveau. En un sens, le présent est simplement ce qui est en ce moment: j’y agis, j’y pense, j’y ressens des émotions, rien n’y est neuf, puisque rien d'autre ne s'y loge que ce qui s'y trouve. Le futur, que j'en espère beaucoup ou que j'en désespère constamment, ne peut que m’offrir des présents successifs: ce présent-ci, puis ce présent-là et cet autre encore, etc. Le futur ne réserve pas de nouveauté, car à chaque fois que je saute en lui, j’y découvre du 14 présent, rien que du présent. Tandis que le passé est continu en moi, il ne cesse de m’habiter. En quoi réside sa nouveauté? Justement en ce que chaque saut dans le futur, pour y investir le présent qui est le mien maintenant, modifie le passé. Le présent est donc plus complexe qu'il ne le semble, puisque, dans chaque présent, s'installe un déphasage entre la sensation étroite du moment qui fuit, n'apparaissant que ce qu'il est, et ce sentiment sans cesse croissant du passé que je reconsidère et qui est toujours plus ce qui m’apparaît.

Mon rapport au futur est discontinu, mon rapport au passé continu : quant au présent, il articule, dans un dédoublement chronique, le continu et le discontinu. C'est pourquoi seul le passé peut m'offrir sans cesse de nouvelles surprises. C'est pourquoi ce que je voudrais savoir porte bien sur le passé, dans la mesure où il m’est à chaque fois offert sous des jours inédits par mon présent. L'anomie ou la dépression s'installent lorsque le passé n'offre plus de surprises parce qu'en réalité mon présent est vide et mon futur inopérant; alors le passé devient un fardeau et un manque au lieu d'être une richesse et un désir.

On dira peut-être que mon sentiment habituel est pourtant de trouver des surprises dans ce que le futur me réserve; mais c'est croire que le futur est toujours déjà là, tout prêt, derrière la porte et qu'il m'attend dans le noir pour me faire peur ou pour me faire tomber un seau d'eau sur le crâne comme un gamin farceur. L'impression d'étonnement vient en fait de ce que mon passé ne semblait justement pas conduire linéairement à ce qui m'arrive ; ce qui m’oblige, du coup, à le reconsidérer pour y réunir ce qui en est apparemment tout à fait délié: de là ce frisson frivole de la surprise. Ce n'est pas le futur qui m’étonne dans le présent qui y mène; c'est le passé qui me surprend dans le futur que je découvre. Voilà pourquoi, dans le présent, cohabitent la contingence de ce qui arrive et l'inter- 
prétation qui en fait un événement rattaché à tout mon passé. De ma puissance d'interprétation, ou plus exactement d'expérimentation, dépend la richesse de ce passé en moi. Mais cette puissance est toujours un rapport de puissances et implique l'héritage de ce que je ne suis pas. Penser la situation comme un pli du temps dans lequel dispositifs sensibles et dispositions intelligibles sont contractés est justement ce que l'intermédialité devrait prendre en charge.

Du concept d'intermédialité, reste à analyser l'autre élément: le médium. Même s'il a pour vocation, bien souvent, de disparaître et de s'effacer sous le message qu'il transmet, il ne saurait être plus neutre que son préfixe. De même que le signe ne parvient à jouer son rôle qu'en mettant de l'avant sa matérialité tout en cherchant à la faire oublier, le médium opère simultanément comme un appareil de transmission indispensable et comme le simple prolongement sans conséquence d'un quelconque outillage mental. C'est précisément la découverte de cette simultanéité à partir du cinéma ou de la peinture cubiste (on oublie souvent ce contexte) qui amène Marshall McLuhan à lancer sa fameuse formule:

Le message du médium cinéma, c'est le passage des connexions linéaires à la configuration. [...] Le cubisme substitue au «point de vue» ou à l'illusion de la perspective une vision simultanée de toutes les faces de l'objet. [...] N'est-il pas clair qu'au moment où le séquentiel le cède au simultané, nous passons dans un monde de structure et de configuration? N'est-ce pas ce qui s'est produit en peinture, en poésie et dans le domaine des communications? Des segments spécialisés d'attention ont disparu au profit de la totalité du champ, et nous pouvons désormais dire le plus naturellement du monde: «le message, c'est le médium ${ }^{4}$.

À compter du moment où la configuration prend en compte le pliage dans un même temps de contenus et d'appareils de médiations, le message se voit, en effet, immédiatement relié à cette double instance et prend tout son sens par l'effet d'immédiateté fabriqué par le médium. C'est à force d'évidence que le médium disparaît en général dans l'attention portée aux contenus, car il ne se situe pas simplement au milieu d'un sujet de perception et d'un objet perçu, il compose aussi le milieu dans lequel les contenus sont reconnaissables et déchiffrables en tant que signes plutôt qu'en tant que bruits. Le médium rend accessible à tous, en même temps qu'il expose à chacun, les contenus ou les formes nécessaires pour la vie en société. De même que l'être-entre (inter-esse) compose des sujets à relier, le médium a pour vocation un rapport au public: «in medium

4. Marshall McLuhan, Pour comprendre les médias: Les prolongements technologiques de l'homme, trad. Jean Paré, Montréal, Bibliothèque québécoise, 2001 [1966], p. 44-45. 
vocare » (littéralement, appeler au milieu) signifie, pour les Latins, «soumettre au jugement public». Le médium est donc ce qui permet les échanges dans une certaine communauté à la fois comme dispositif sensible (pierre, parchemin, papier, écran cathodique sont des supports médiatiques) et comme milieu dans lequel les échanges ont lieu.

On peut prendre l'exemple antique des cités grecques qui avaient chacune leur autonomie politique, mais aussi leurs coutumes particulières et leurs rituels spécifiques. Or, à ce mouvement centrifuge répond aussi un mouvement centripète par où une culture hellénique se dessine (pour mieux éviter un État central unique) qui passe par des manifestations communes: lors des jeux Olympiques ou des Panathénées, des concours permettent de sélectionner parmi les performances orales (péans apolliniens, dithyrambes dionysiaques, chants de banquet, poèmes homériques) celles qui semblent exemplaires d'une culture qu'on invente. Cette culture permet de composer un milieu où chacun puisse à la fois reconnaître sa spécificité et apprécier son universalité. Il est significatif que le verbe hellenizein désigne autant le fait de parler Grec, que de parler correctement ou même de parler tout court. Dans ce rassemblement de sens pointe l'oubli qu'une langue n'est pas tout le langage. Mais hellenizein signifie aussi le fait d'être Grec, d'être un homme digne de ce nom, voire d'être un homme tout court: le médium du langage se replie impeccablement sur la dimension d'appartenance à une communauté jusqu'à l'être même de l'homme. Les poèmes homériques chantent justement ce destin commun des Grecs et les rhapsodes des diverses cités tentent de coudre à leur manière les multiples passages des épopées afin de glisser leurs singularités dans l'histoire générale de la Grèce. Quand Hipparque d'Athènes, au $\mathrm{VI}^{\mathrm{e}}$ siècle avant J. C., fait enfin mettre par écrit les poèmes, la culture commune est devenue loi de tous.

De même, la tragédie, d'abord propre à Athènes, devient non seulement un montage « intermédial » (danse, pantomime, musique et déclamation), mais aussi un rassemblement panhellénique, absorbant genres de performance (des chansons de banquet aux lamentations funèbres) et mémoires des diverses cités grecques, placé sous la figure nouvelle d'un «auteur ». Là où les aèdes ne performaient leurs chants qu'en se donnant comme les supports de la voix des Muses, les auteurs tragiques rapportent à eux-mêmes l'invention écrite des mythes communs. Du coup, l'énonciation réelle de la performance de l'aède se transforme en énonciation fictive qui prend en charge, dans un tout autre contexte d'énonciation (le concours et le festival), des énoncés préexistants. Plus encore que la coupure entre oralité et écriture, c'est cette dissociation et réinscription de 
l'énoncé et de l'énonciation qui engendrent à la fois une séduction nouvelle et une prudente crainte ${ }^{5}$. Platon cherche à contrôler ces dangers par une double tactique: d'un côté, le Ion lui permet de montrer le rhapsode comme incapable de savoir vraiment ce qu'il dit (ses qualités d'énonciateur ne le conduisent pas à comprendre vraiment les énoncés), d'autre part, l'enjeu proprement politique de la condamnation de la mimesis dans la République l'autorise à rejeter la fictionnalité au profit d'une éducation plus philosophique qui en déchiffrerait les contenus de vérité. Aristote, qui a l'air au contraire de valider le principe de la mimesis, trouve en fait dans sa Poétique et dans sa Rhétorique une autre manière de contrôler les productions publiques des passions communes, en les codifiant et en réduisant le spectaculaire à des effets sans portée générale. C'est tout un nouveau milieu de production des énoncés de vérité qui s'articule ainsi à la construction d'une culture commune (sans qu'on aperçoive aisément son édification), en même temps qu’à la révélation ou à l'effacement de la matérialité de certains supports. On voit donc dans ce cas de figure combien il est nécessaire de lier l'élaboration des idées à une construction du public et l'histoire de la vérité à l'invention et à la diffusion des supports.

Si le médium semble simplement transmettre un message selon le schéma du télégraphe devenu un moment classique dans les théories de la communication: émetteur-message-récepteur, il s'agit en fait de rappeler qu'il faut aussi prendre en compte l'opacité propre à chaque médium et, surtout, les configurations spécifiques de sens et les jeux de pouvoir particuliers qu'il implique. Qui maîtrise les techniques, qui en autorise les effets, qui en assure la diffusion compte autant que l'auteur de telle ou telle œuvre, dès lors que l'on considère que les significations ne transitent pas d'un cerveau à un autre de façon immatérielle et immédiate à l’image de la communication angélique ${ }^{6}$. On ne saurait comprendre et s'approprier de la même manière le Bourgeois gentilhomme de Molière si l'on assiste à sa représentation au sein de la grande fête de cour que donne Louis XIV

5. Sur ces points, voir Gregory Nagy, Pindar's Homer. The Lyric Possession of an Epic Past, Baltimore, Johns Hopkins University Press, 1990; Marcel Détienne, Les maîtres de vérité dans la Grèce archaïque, Paris, Éditions La Découverte, 1990; Florence Dupont, L'invention de la littérature: De l'ivresse grecque au livre latin, Paris, Éditions La Découverte, 1994 .

6. Voir Donald McKenzie, Bibliography and the Sociology of Texts, Londres, The British Library, 1986; Roger Chartier, Culture écrite et société: L'ordre des livres (XIV-XVIII siècle), Paris, Éditions Albin Michel, 1996. 
à Chambord le 14 octobre 1670 , ou à sa reprise à Paris pour le public de la ville le 23 novembre, ou plus encore si on le lit dans l'édition de la Pléiade (où la fragilité du papier bible et le luxe de la reliure ajoutent au soin des notes le sentiment d'un grand texte) ou dans une édition scolaire du genre des Classiques Larousse (où le mauvais papier et la reliure bon marché accroissent l'effet contraignant des questions d'apprentissage). Ce sont à chaque fois des affaires d'appareil, donc d'apparences: appareil spectaculaire de l'État, appareil érudit de notes, appareil scolaire de l'ennui.

Les matérialités de la communication font ainsi partie intégrante du travail de signification et d'interprétation des contenus ${ }^{7}$. On ne peut faire l'économie de la technologie impliquée dans tout médium: elle joue, au contraire, un rôle important dans les manières d'appréhender et de construire du sens, même si elle doit disparaitre sous ce qu'elle permet de transmettre. Le logos a ainsi longtemps été perçu - ou plutôt construit - non seulement comme supérieur à la techné, mais encore comme indépendant de la technique. Il faut, à l'inverse, redonner sa double valeur au terme de «techno-logie » et saisir combien c'est la technique qui a permis les ressources du logos (qu'on le traduise - opération de transmission qui, elle-même, contraint la réception de ce qui est dit! - en ouvrant son Bailly par raison, langage, parole, fable, bruit, réputation, histoire, livre, conversation, argument ou compte...). Du point de vue paléontologique, André Leroi-Gourhan a montré que la station debout avait libéré la main, comme organe de capture, et que la main-outil avait libéré la bouche, comme organe de communication (que ce soit la parole ou le baiser): autrement dit, le langage naît avec la technique ${ }^{8}$. Cela a pour conséquence que des bouleversements dans les médias de communication changent aussi les modalités de la pensée et la fabrication du sens. Ainsi l'invention de l'écriture ou son expansion par l'imprimerie ont-elles à l'évidence changé certaines façons de raisonner et certaines constructions de valeurs?.

Il ne s'agit pourtant pas simplement de reporter le poids des significations sur les bouleversements technologiques, mais de faire jouer les médiations dans tous

7. Voir Materialities of Communication, Hans Ulrich Gumbrecht, Ludwig Pfeiffer (éds.), trad. William Whobrey, Stanford, Stanford University Press, 1994.

8. André Leroi-Gourhan, Le geste et la parole: technique et langage, Paris, Éditions Albin Michel, 1964.

9. Voir Jack Goody, The Domestication of the Savage Mind, Cambridge, Cambridge University Press, 1977; Elizabeth Eisenstein, The Printing Revolution in Early Modern Europe, Cambridge, Cambridge University Press, 1983. 
les sens: une modification des supports de transmission des savoirs joue sur la construction des significations, mais de telles modifications sont aussi les effets d'idées qui en ordonnent les puissances singulières. L'idée est une contraction de cas empiriques singuliers; les pratiques médiatiques des diffusions et des vaporisations d'idées. Il faut donc penser une continuité, qui suppose une différence de degré et non de nature, entre l'intelligible et le sensible ou entre le logique et l'empirique. Si nous donnons à un énoncé logique une valeur intemporelle, ce n'est pas que nous croyons forcément à son éternité, mais que nous avons besoin de figer certains énoncés afin de mieux appréhender la circulation et la variabilité comparée des autres énoncés. Ainsi, «tout être humain a des parents » sonne comme une proposition logique dont on aurait du mal à concevoir le contraire (ce qui peut fonctionner comme un critère de logicité d'un énoncé); sauf à prendre en compte le caractère social plus que biologique de la parenté (ce qui nous ferait comprendre la valeur des relations avunculaires dans certaines sociétés sauvages) ou les nouveautés que la biotechnologie permet aujourd'hui (ce qui nous ouvrirait à une autre considération des bébés-éprouvettes). Un énoncé logique peut donc toujours être redistribué dans la circulation usuelle des discours et retrouver une historicité dont il semblait avoir été absous. C'est ainsi que l'on peut produire une «histoire de la vérité » ou une «archéologie des a priori».

Toutes ces médiations ont à chaque fois des histoires: les intermédialités en forment les plans de consistance. On ne peut, par conséquent, analyser uniquement les rapports de la gravure, de l'écriture (en deux langues) et de la musique dans le cas de cette belle page du livre de Michael Maier, Atalanta fugiens ${ }^{10}$. Le titre lui-même nous invite, bien sûr, à multiplier les relations puisque ces «emblèmes chimiques des secrets de la nature» doivent toucher autant les yeux et l'intelligence que les oreilles et l'âme, suscitant ainsi « un plaisir singulier à voir, lire, méditer, comprendre, juger, chanter et entendre». Mais ces différents registres artistiques apparaissent convocables dans un seul ouvrage, dans la mesure où, surtout, s'y cristallisent simultanément quête mystique, investigation scientifique, héritages mythologiques, dispositifs techniques, opération commerciale et

10. Michael Maier, Atalanta fugiens, hoc est, Emblemata nova de secretis naturce chymica, accomodata partim oculis \& intellectui, figuris cupro incisis, adjectisque sententiis, epigrammatis \& notis, partim auribus \& recreationi animi plus minus 50 fugis musicalibus trium vocum, quarum duce ad unam simplicem melodiam distichis canendis peraptam, correspondeant, non absq[ue] singulari jucunditate videnda, legenda, meditanda, intelligenda, dijudicanda, canenda 6 audienda, Oppenheim, Jean Theodore de Bry, 1618. 


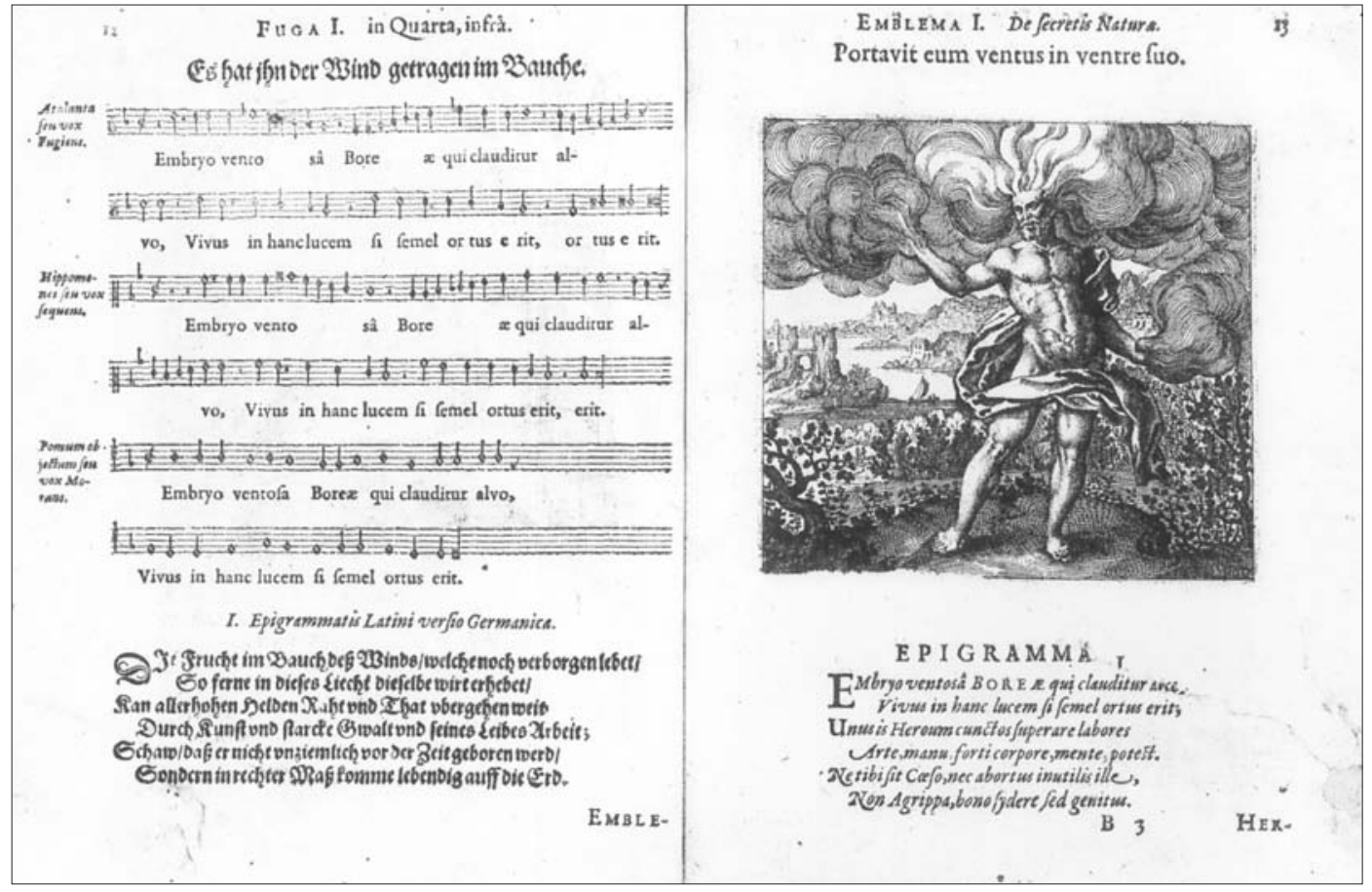

- Michael Maier, Atalanta fugiens, 1618 
stratégie politique. En effet, cet ouvrage participe du soudain et bref essor du mouvement des Rose-Croix en Allemagne, où se mêlent nouveaux savoirs médicaux et chimiques, anciens prestiges de l'hermétisme, réforme religieuse et alliances politiques du moment entre l'Électeur palatin et le roi d'Angleterre. Mais il faut aussi compter sur la puissance de libraires comme la famille de Bry et la qualité supérieure de la gravure sur cuivre récemment répandue pour comprendre toute la portée de ces publications ${ }^{11}$.

Bref, le premier emblème de ce livre à la facture très soignée ne fait pas que reprendre un fameux vers d'Hermès Trismégiste invoquant le souffre (feu ou esprit de la pierre philosophale) que Mercure, divinité fuyante comme le vent, porte en son ventre, il permet, dans le paradoxe d'un corps d'homme enceint d'un enfant, l'association de la matière et de l'esprit. Ce sont tous ces éléments dont la dynamique forme un canevas dont l'intermédialité doit rendre compte.

De façon exemplaire, on pourrait voir à l'œuvre dans le merveilleux roman de Balzac, Illusions perdues, la traversée fictionnelle d'une situation intermédiale. Il ne s'agit pas simplement, dans cet ouvrage, de retracer le destin d'un poète, «grand homme de province » monté à Paris et faisant la douloureuse épreuve de sa vanité. Balzac met, en fait, en scène l'ensemble des dispositifs matériels (depuis la fabrication du papier et le travail de l'imprimerie ou des journaux jusqu'à la circulation des imprimés et la vente en librairie) et des usages du travail immatériel des poètes, romanciers, journalistes ou érudits, en passant par les puissances institutionnelles du salon, de la loge d'opéra, du clan d'amis d' "avant-garde », de la critique littéraire, des éditeurs et des libraires, ainsi que des juristes pour les brevets et les procès. Ce sont ces ensembles instables de rapports de puissance qui forment le réseau d'intermédialités dans lequel pratiques littéraires et désirs d'écriture trouvent leurs valeurs et leurs sens. Les illusions perdues ne sont pas seulement celles du jeune poète, mais aussi bien celles du lecteur qui découvre comment les idées novatrices ou ringardes sont toujours prises dans des techniques, des habitudes, des institutions et des lois.

11. Sur ces questions, voir Frances Yates, The Rosicrucian Enlightenment, Londres, Ark Paperbacks, 1986 [1972], p. 80-102; Stanislas Klossowski de Rola, Le Jeu d'Or: Figures hiéroglyphiques et emblèmes hermétiques dans la littérature alchimique du XVII siècle, Londres, Éditions Thames \& Hudson, 1997 [1988], p. 59-104; Roland Edighoffer, Les Rose-Croix et la crise de la conscience européenne au XVII siècle, Paris, Éditions Dervy, 1998, p. 125-161. 
Maintenant que voici précisés l'«inter» et les «médias», on pourrait se demander où réside la nécessité de redoubler de la sorte le principe d'un entredeux. Puisque la médialité désigne ce qui est au milieu, pourquoi ajouter encore « inter », comme l' «entre de ce qui est au milieu »? Pareil redoublement renvoie, en fait, au déphasage même de l'instant dans lequel s'articulent dispositifs sensibles et dispositions intelligibles, singularités de l'événement et règles idéelles, forces matérielles et signes de pensées. La médialité étant ce milieu dans lequel les forces se transforment en signes et les idées en pouvoirs grâce à des institutions, le préfixe attire l'attention sur ces doubles jeux constants des phénomènes de l'histoire. Mais il faut encore souligner un troisième pli du concept, puisque le suffixe, cette fois, impose une autre sorte de redoublement en faisant référence au fait ou au caractère d'être ceci ou cela. Comme l'inanité désigne le fait d'être vain, l'intermédialité renvoie au caractère de ce qui se trouve entre des milieux. Le suffixe ajoute un élément de réflexivité supplémentaire qui rend encore plus visible les effets d'immédiateté des milieux et les articulations qui les composent ou qui les hantent.

Le concept d'intermédialité opère alors à trois niveaux différents d'analyse. Il peut désigner, d'abord, les relations entre divers médias (voire entre diverses pratiques artistiques associées à des médias délimités): l’intermédialité vient ainsi après les médias. Ensuite, ce creuset de différents médias d’où émerge et s'institutionnalise peu à peu un médium bien circonscrit: l'intermédialité apparaît, dans ce cas-ci, avant les médias. Enfin, le milieu en général dans lequel les médias prennent forme et sens : l'intermédialité est alors immédiatement présente à toute pratique d'un médium. L’intermédialité sera donc analysée en fonction de ce que sont des «milieux» et des «médiations", mais aussi des «effets d'immédiateté», des «fabrications de présence» ou des «modes de résistance». On discerne sans doute la proximité de ce genre d'analyse avec les travaux inspirés par Régis Debray et la médiologie. Deux différences sont néanmoins notables. D’une part, les intermédialités renvoient à de modestes configurations historiques, par définition variables, et non à une science des médiations, dont le fréquent souci du détail n'a d'égal que les vastes classements aux synthèses rapides ${ }^{12}$. D'autre part, héritant volontiers de l'histoire des mentalités (tout en produisant une archéologie de la vitesse plutôt qu'une histoire de la lenteur), et plus généralement d'un

12. Voir, par exemple, le tableau des trois sphères qui recense toutes les formes sociales des médiations depuis l'invention de l'écriture: Régis Debray, Cours de médiologie générale, Paris, Éditions Gallimard, 1991, p. 534-535. 
modèle herméneutique, la médiologie cherche sous les effets de surface l'être caché des profondeurs $^{13}$; alors que c'est immédiatement et par des effets latéraux que se nouent et se contractent les intermédialités dans une situation.

S'il en va bien de la sorte, il n'est pas possible de fournir seulement comme ancrage du concept d'intermédialité quelques variations intellectuelles sur l'inter ( - texte, - discours ou - média), puisque ce serait le réduire à de simples configurations d'idées hors de toute matérialité et de tout milieu. Il y a, bien entendu, une certaine vie des concepts et il est possible d'en établir des généalogies, mais ils sont pris dans des schèmes organisateurs qui en permettent les façons ou les transformations, des formations historiques qui en légitiment les pratiques et en valorisent les usages, des modes sociaux qui en alimentent les productions et les reconnaissances. Il faudrait donc pouvoir reconstituer ces milieux de sens afin de mieux saisir les implications du concept d'intermédialité. Comme ce serait trop long pour les dimensions modestes de mon propos, je me contenterai d'indiquer un de ses soubassements, d'autant plus important qu'on l'y relie rarement: le développement du travail immatériel. Il y a, en effet, quelque chose à penser du rapport entre, d'une part, la valeur allouée à la relation, et au passage des matériaux, des forces et des techniques aux messages et aux idées et, d'autre part, l'emprise récente du travail que l'on dit, depuis l'économie politique classique, «improductif» ou «immatériel».

Avec l'essor du capitalisme, il devenait manifestement important d'établir des modes scientifiques de calcul des richesses: plutôt que la dextérité utile de l'artisan ou l'usage agréable de biens ou de relations, c'était le temps de travail qui allait permettre d'établir une unité de calcul. Ainsi, pour Adam Smith, le travail productif stocke du temps dans la marchandise et fait alors la valeur des biens; tandis que le travail, qu'il baptise «improductif», dépense du temps dans des performances et fait la valeur des «services» (comme les nomme par la suite Malthus, réservant la notion de «travail» pour la seule production des marchandises). Le travail devient une valeur sociale, là où il désignait anciennement la

13. «La télédétection par satellite sait à présent établir une corrélation entre le mouvement des vagues en surface et le relief des fonds océaniques. Ainsi le médiologue détecte par la surface les profondeurs d'un courant de pensée, d'une famille d'esprit, d'une mouvance.» Régis Debray, Cours de médiologie générale, p. 78. Plutôt que l'histoire des mentalités, c'est bien plutôt l'histoire du livre qui fournirait l'inspiration de ce type d'investigation. 
dépossession de soi et l'aliénation dans une contrainte servile ${ }^{14}$. On peut aussi noter que le concept de travail trouve justement sa formulation en physique au moment où il est valorisé dans l'économie politique: analysant l'homme comme une machine, Coulomb puis Coriolis dégagent les principes du calcul de quantités d'action-travail, comme Say et Ricardo font du travail l'instrument central du calcul des productions d'utilité ${ }^{15}$.

Le taylorisme qui gagne l'industrie triomphante du début du $\mathrm{xx}^{\mathrm{e}}$ siècle est le prolongement de cette double dimension économique et physique du travail: l'étude expérimentale de la machine humaine permet d'en calculer (et donc d'en maximiser) les énergies nécessaires pour la production de valeur. Ainsi, le savoirfaire scientifiquement décomposé permet de rendre les ouvriers interchangeables, de même qu'en horlogerie, dans ces mêmes années, les pièces inter24 changeables réduisent la variété des productions multiples. Le travail à la chaîne est censé permettre une spécialisation des gestes de l'ouvrier dont on exploite le travail dans sa seule fonction mécanique, afin de stocker dans les biens produits un maximum de temps, sans égard pour les savoirs personnels dont il disposerait. Or, c'est justement sur ce point que s'est fait le passage entre le modèle fordiste de l'industrie (qui repose sur le taylorisme) au modèle «toyotiste» ou «post-fordiste» qui invite à utiliser des professionnels polyvalents dont les énergies propres et les savoirs personnels sont mobilisés pour les fins du travail collectif de l'usine: l'ouvrier accomplit moins un programme détaillé à l'avance qu'il ne fournit une prestation globale jugée en fonction de son résultat. Cette exploitation des services plus encore que des productions matérielles ne touche pas simplement l'industrie (depuis l'ouvrier qualifié jusqu'à l'enchaînement des biens et des services: après-vente, crédit, etc.), mais l'ensemble de la société où le secteur tertiaire gonfle rapidement dans l'après-guerre. Le développement technique des nouvelles formes de communication et l'insistance sur la circulation de l'information comme capital accroissent encore la valeur du travail immatériel dont l'«improductivité » apparaît de plus en plus rentable. Même si l'industrie demeure enco-

14. Voir Marshall Sahlins, Age de pierre, âge d'abondance, Paris, Éditions Gallimard, 1976; Jean-Pierre Vernant, Pierre Vidal-Naquet, Travail et esclavage en Grèce ancienne, Paris, Éditions Complexe, 1988; Robert Fossier, Le travail au Moyen Âge, Paris, Éditions Hachette, 2000.

15. Voir François Vatin, Le travail: Economie et physique, 1780-1830, Paris, Presses universitaires de France, 1993. 
re au centre des compétitions nationales et n’a pas été partout dominée par le secteur tertiaire, la relation de service est devenue l'élément clef de l'économie contemporaine et l'employé de bureau le modèle général du travail (au point de déterminer, par exemple, la configuration de tous les ordinateurs, comme si, quoi que nous fassions et quel que soit l'usage que nous réservions à nos ordinateurs, il nous fallait adopter la disposition matérielle, cognitive et sociale du bureau).

Là où l'ouvrier vendait sa force de travail comme quelque chose qu'il possédait et dont il se trouvait, du coup, aliéné en devenant machine, l'homme de services échange, avant tout, ce qu'il est et qu'il doit constamment renouveler à la manière d'un surhomme. Comme toujours, les jeux collectifs, en particulier télévisés, sont particulièrement instructifs des nouveaux régimes de production : aux jeux de force ou de savoir, que les concurrents possèdent et exhibent, succèdent de plus en plus des jeux où les concurrents valent pour ce qu'ils sont et se trouvent ainsi échangés comme valeurs ou rejetés comme pertes - c'est ainsi leur vulnérabilité personnelle qui est mise en jeu ${ }^{16}$. Cela ne signifie pas qu'il faudrait regretter le bon vieux temps des usines Ford, mais que de nouvelles formes d'aliénation se sont mises en place au nom même de l'autonomie et de la singularité des individus. Il faut en particulier prendre garde à ce que ces échanges, fondés sur le caractère singulier et vulnérable des êtres, puissent trouver dans la blessure et dans la faille les valeurs propices de l'ouverture et de la mémoire ${ }^{17}$.

Dans la mesure où la performance industrielle est moins liée à une sectorisation des activités (productivité au poste de travail) qu'aux nouvelles valeurs de $l^{\prime}$ «écosystème relationnel ${ }^{18}$ » (productivité globale de l'usine) et des technologies de la communication en plein essor, le travail immatériel devient une incontes-

16. Loft story, Le maillon faible, Kolantha offrent ainsi le spectacle de soi et la scénographie de l'exclusion comme d'impeccables retours de l'exclusion sociale et de la demande de services; ou encore, tel jeu australien (pour le moment encore refusé en Amérique ou en Europe), qui calcule le taux de stress d'un concurrent soumis aux questions agressives d'un présentateur, donne une bonne image du stress accru des employés qu'on a rendus responsables du résultat collectif de l'entreprise.

17. Sur le rapport de la mémoire au vulnérable et à la blessure, je me permets de renvoyer à nouveau à mon ouvrage De l'immédiateté, notice 4.

18. Pierre Veltz, Des lieux é des liens: Politiques du territoire à l'heure de la mondialisation, La Tour d'Aigues, Éditions de l'aube, 2002, p. 85. Sur ces questions, voir plus généralement les ouvrages d'André Gorz, Métamorphoses du travail. Quête du sens: Critique de la raison économique, Paris, Éditions Galilée, 1988, et Misères du présent. Richesse du possible, Paris, Éditions Galilée, 1997. 
table source d'énergie sociale. Pour les Grecs, la liberté était parole; pour nos contemporains, la parole est travail. On peut certes en espérer la production d'une «intelligence générale ${ }^{19}{ }$, mais le travail immatériel se voit plus valorisé dans sa dimension statistique (je ne m’échange pas dans mes goûts les plus singuliers, bien plutôt dans la communicabilité même de mon être), voire, au bout du compte, impersonnelle (d'où des emplois de la terreur qui ne frappent plus des adversaires caractérisés, mais des images-types). Les goûts ne sont importants pour le système d'information qu'en ce qu'ils permettent aux industries de travailler à «flux tendus»: alors que la production fordiste cherche dans la programmation à large échelle une rentabilité des stocks, au contraire, dans les nouveaux modes industriels, l'opportunité, passant par l'information, tente de raréfier les stocks; d'un côté, la production publicisée régit la consommation, de l'autre, la consommation déchiffrée engendre la production. Cela a pour effet de rendre le travail encore plus contraignant, sous des dehors libérateurs, en forçant les travailleurs à rester toujours plus disponibles afin de saisir les occasions incessantes que l'information produit $^{20}$. Le flux tendu, c'est l'immédiateté mise au service du capital.

On comprend donc que l'impact du travail immatériel sur les conditions d'existence ne soit pas un sauf-conduit pour le paradis des nourritures spirituelles (ni même matérielles). Il faut, en fait, voir jouer les résistances dans les lieux mêmes où s'exercent les contraintes: vulnérabilité, immédiateté ou opportunité (le sens d'un certain kairos) peuvent ainsi devenir de justes forces plutôt que d'illusoires valeurs. Au stockage de temps dans la marchandise, qui suppose la temporalité pleine, linéaire et extensive de la production matérielle, doit s'opposer une autre vertu de la production immatérielle: la projection de temps dans la matière et son usage, impliquant un temps dynamique, lacunaire et intensif. Car il ne faut pas se tromper sur l'immatérialité: elle n'implique pas simplement un royaume des Idées loin des contingences humaines, mais une autre manière de

19. Sur les valeurs de cette notion, voir André Tosel, «Centralité et non-centralité du travail ou la passion des hommes superflus ", La crise du travail, Jacques Bidet, Jacques Texier (dirs.), Paris, Presses universitaires de France, 1995, p. 209-218; Michael Hardt, Antonio Negri, Empire, trad. Denis-Armand Canal, Paris, Exils Éditeur, 2000; Paolo Virno, Grammaire de la multitude: Pour une analyse des formes de vie contemporaines, trad. Véronique Dassas, Nîmes, Montréal, Éditions de l'éclat, Conjonctures, 2002.

20. Sur cette question et généralement sur la place du langage dans cette nouvelle économie, voir Christian Marazzi, La place des chaussettes: Le tournant linguistique de l'économie et ses conséquences politiques, trad. Anne Querrien, François Rosso, Paris, Éditions de l'éclat, 1997. 
prendre en compte la matière dans son rapport au temps. Cela permet alors de récupérer non seulement une valeur d'échange, mais aussi une valeur d'usage des êtres, c'est-à-dire une puissance et une jouissance de soi et des autres.

Telle est la situation sociale dans laquelle intervient la notion d'intermédialité, issue de cette vocation aux échanges, alimentant cette fin d'une clôture de la production des marchandises sur elles-mêmes, jouant de cette valorisation du travail immatériel et des procédures symboliques du langage. Telle est en même temps la chance qu'elle nous offre. Loin de rester dans la sphère mystérieuse d'esprits immatériels, c'est justement la résistance même des matières et des temps, des médias et des situations, dont elle fait l'épreuve dans l'usage des relations, des choses et des personnes. Sa dimension d'immédiateté permet de repenser, voire de conserver dans la dynamique des valeurs purement marchandes des flux tendus et des productions de présence certains restes incertains, des restes anachroniques ${ }^{21}$.

Toute culture a beau opérer nécessairement en fonction d'effets d'immédiateté, elle le fait selon des circuits médiatiques différents, selon des arts et des techniques qui se modifient ou se rassemblent: l'intermédialité en élabore certains plans de consistance dont il s'agit de rendre compte et, surtout, de voir articulées les illusions régulatrices et les résistances opportunes. Ce n’est pas simplement une vertu démystificatrice de ce qu'on appelait «idéologie», mais le double sens ou le double jeu de l'illusion et de la perte. En ce sens, l'intermédialité n’offre pas un «dépassement» des anciennes intertextualités ou interdiscursivités: elle insiste simplement sur ce qui fondait ces concepts, c'est-à-dire le sens privilégié alloué aux enchaînements, aux mouvements de désappropriation et d'appropriation, aux continuités tacites ou affirmées, aux restes obstinés et aux recyclages diserts. Pour le dire d'un mot, elle nous fait perdre du temps avec bonheur dans un monde qui voudrait toujours en gagner.

21. L'enjeu politique du reste, de la résistance et de la temporalité lacunaire de l'anachronos ou du kairos (autrement dit, de l'opportunité) comme non-coïncidence avec soi est explicitement thématisé par Giorgio Agamben dans Le temps qui reste: Un commentaire de l'Épître aux Romains, trad. Judith Revel, Paris, Éditions Rivages, 2000, p. 90-97. Voir aussi Françoise Proust, De la résistance, Paris, Éditions du Cerf, 1997. À titre d'exemple, on pourrait trouver, du côté littéraire, dans la coïncidence immédiate d'une balle et d'une idée, telle que la met en scène Pierre Ouellet, une dimension proprement intermédiale de son détective amnésique et du statut du savoir : «On l'a retrouvé dans la rue, sans connaissance. Une balle dans la tête. Ou l'idée même de la mort dans la boîte cranienne, qui l'a effleuré. » Still. Tirs groupés, Québec, Éditions L’instant même, 2000, p. 16. 